\title{
VERZEICHNIS DER AUTORINNEN UND AUTOREN LES AUTEURS DE CE LIVRE
}

Fanny COSANDEY, maître de conférences à l'École des hautes études en sciences sociales (EHESS), Paris

Denis CrouzeT, professeur d'histoire moderne à l'université Paris IVSorbonne

Leonhard HOROWSKI, wissenschaftlicher Assistent an der Technischen Universität Berlin

Achim LANDWEHR, Juniorprofessor für Europastudien an der Heinrich-HeineUniversität Düsseldorf

Armelle LEFEBVRE, boursière francophone à l'Institut historique allemand Paris (2004-2005)

Wolfgang REINHARD, Emeritus für Neuere Geschichte, Universität Freiburg im Breisgau

Albert RIGAUDIÈRE, membre de l'Institut, professeur d'histoire de droit à l'université Paris II-Panthéon-Assas

Lothar SCHILLING, Privatdozent für Neuere Geschichte an der Universität zu Köln, Gastdozent des Deutschen Historischen Instituts (2004-2005)

Gerrit WALTHER, Professor für Neuere Geschichte an der Universität Wuppertal 
